\title{
Advanced Biomechatronics and Locomotion Laboratory: Robotics for Rehabilitation and Mobility
}

\author{
Mojtaba Ahmadi \\ Mechanical and Aerospace Engineering, Carleton University \\ 1125 Colonel By Drive, Ottawa, Ontario, K1S 5B6, Canada \\ mojtaba.ahmadi@carleton.ca
}

\section{Extended Abstract}

The population is ageing in Canada and healthcare expenses are on the rise, hence requiring novel practices and equipment to increase efficiency and promote patient autonomy. Robotics has shown promise in providing less invasive methods in diagnostic, therapeutic, and surgical procedures and more recently as a rehabilitation tool. In the last decade, several new medical robots have been proposed, prototyped, or commercialized such as Da Vinci robot for surgery, Cyber knife for radiotherapy, and the Locomat system for rehabilitation.

With a short introduction to medical robotics, the newest robotic systems developed at Carleton University's Advanced Biomechatronics and Locomotion (ABL) laboratory will be reviewed. Some of the most pressing issues related to rehabilitation robotics, assistive devices, and safe human-robot interaction are the core research areas highlighted. Damages to the central nervous system, caused by neural illnesses or injuries, may be only addressed via extensive and early rehabilitation conforming to the main principles of motor learning. As such, a novel robotic system, called Virtual Gait Rehabilitation Robot (ViGRR), capable of providing early and intensive gait therapy will be discussed. The system exploit virtual reality or computer gaming to ensure patient engagement. The development of an Omni-directional robotic support systems, called GaitEnable (now commercialized as Solowalk), which enables early mobilization in acute care or rehabilitation departments in hospitals will also be presented. Other novel devices and research results obtained at ABL are highlighted, spanning from sensing and biofeedback techniques for balance aid in elderly, interaction sensing and control for safer human-robot interactions, and assistance regulation techniques for assistive devices, to the general research on dynamics and learning of balance in bipedal walking. 